\title{
LA DENSIDAD Y TAMAÑO DE PLÁNTULAS CON CEPELLÓN EN LA PRODUCCIÓN DE ESPÁRRAGO VERDE ${ }^{1}$
}

\author{
Ana María Castagnino ${ }^{2}$, Karina Elizabeth Díaz ${ }^{2}$, Patricia Sastre Vázquez ${ }^{2}$, María Belén Rosini ${ }^{2}$, Silvina Sasale ${ }^{2}$
}

\section{RESUMEN}

La densidad y tamaño de plántulas con cepellón en la producción de espárrago verde. Con el objetivo de determinar la influencia del tamaño de la celda utilizada para la obtención de plántulas de espárrago y la densidad o marco de plantación empleado, sobre la producción de turiones, se evaluó en invernadero tres tamaños de celda: 1) PG: grande $\left(70 \mathrm{~cm}^{3}\right)$, 2) PM: mediana $\left(30 \mathrm{~cm}^{3}\right)$ y 3$)$ PCh: pequeña (20 $\mathrm{cm}^{3}$ ) y tres densidades (35 714 (D1); 23810 (D2) y 17857 (D3) plantas/ha). Se efectuó un trabajo en la Chacra Experimental de la Facultad de Agronomía, en Azul, provincia de Buenos Aires. La plantación en el campo se efectuó en noviembre 2002, setenta días después de la siembra del almácigo con plántulas con cepellón. La cosecha se realizó del 25/09 al 18/10 del año 2006, en el cuarto año desde la plantación, con una frecuencia de cosecha de día por medio. Se realizó un análisis de la varianza, con un diseño completamente al azar y considerando como factor: el tamaño de las plántulas que provenían de cada contenedor y tres densidades. En el segundo año evaluado, la densidad D1 fue la de mayor producción (4450 kg/ha netos totales) y mayor cantidad de turiones (380 166). Las plantas provenientes de los dos mayores tamaños de celda produjeron $4 \mathrm{t} / \mathrm{ha}$ netas en promedio. Las bandejas de siembra de tamaño G y D1 resultaron más apropiados para la producción de espárrago ya que permitieron obtener el mayor rendimiento en peso y número, y obtener un mejor aparato radical.

\begin{abstract}
Density and size of seedlings with ball of soil on production of green asparagus crops. In order to evaluate the effect of transplanting greenhouse-grown seedlings with a ball of soil in three cell sizes: 1) PG: big $\left(70 \mathrm{~cm}^{3}\right)$; 2) PM: medium $\left(30 \mathrm{~cm}^{3}\right)$ and 3) $\mathrm{PCH}$ : small $\left(20 \mathrm{~cm}^{3}\right)$ and three densities: 35714 (D1); 23810 (D2) and 17857 (D3) pl/ha. The trial was carried out on the experimental unit of the Facultad de Agronomia in Azul, province of Buenos Aires. Field planting was done in November 2002, 70 days after the sowing of the nursery bed with seedlings with ball of soi. Harvest was carried out from 25/09 to $18 / 10$, in the fourth year since planting with a harvesting frequency of every other day. An analysis of variance was performed with a completely randomized design, and considering the seedling size and the three densities as factors. The best density in the second year of evaluation was D1 (4450 kg/ha net totals and 380166 turions). The plants from the two bigger cell sizes produced $4 \mathrm{t} / \mathrm{ha}$, on average. Sowing trays G and D1 were more appropriate for the production of asparagus since they corresponded to the greatest yield in weight and number, and a better root system, essential in a perennial crop.
\end{abstract}

Key words: Seedbed, density of sowing, calibers of turions, Asparagus officinalis L.

Palabras clave: Almácigo, densidad de siembra, calibres de turiones, Asparagus officinalis L.

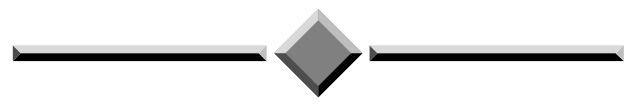

\footnotetext{
Recibido: 30 de noviembre, 2009. Aceptado: 16 de mayo, 2011. Proyecto "Estudio de los puntos críticos de la cadena agroalimentaria espárrago: estrategias para su optimización" del Programa de Investigación y Transferencia Tecnológica "Cadena Espárrago bajo un enfoque sistémico".

2 Centro Regional de Estudio de Cadenas Agroalimentarias y Programa Institucional de Alimentos - Facultad de Agronomía, Universidad Nacional del Centro de la Provincia de Buenos Aires. CC47 (7300) Azul. Argentina; Tel/Fax: 2281 433291/93. amc@faa.unicen.edu.ar, kardiaz@faa.unicen.edu.ar, psastre@faa.unicen.edu.ar, belenrosini@yahoo.com.ar, silvinasasale@yahoo.com.ar
} 


\section{INTRODUCCIÓN}

La producción de espárrago constituye una alternativa de alto valor agregado. Es un cultivo perenne que tiene un período promedio de cosecha de diez años y llega al máximo de producción a los cuatro o cinco años, en el que es necesario considerar todos los aspectos tendientes al logro de un óptimo rendimiento productivo y turiones de elevada calidad. Uno de dichos aspectos está constituido por el sistema de inicio y la necesidad de seleccionar un adecuado marco de plantación.

Para este cultivo se han difundido dos sistemas: el tradicional de arañas (STA) y el moderno de plántulas con cepellón (SMP). En el primer caso se requiere un año para la producción de las coronas. El segundo sistema (SMP), permite optimizar el empleo del espacio disponible para el almácigo y realizar una mejor planificación de las plantaciones definitivas.

El inicio por plántulas producidas mediante empleo de sustratos como vermiculita, perlita y/o turba, presenta otras ventajas que las ya mencionadas como la mayor seguridad sanitaria, ya que se evitan las numerosas heridas provocadas a las raíces y a los rizomas durante la extracción de arañas, las que constituyen una fácil vía de penetración de hongos patógenos.

El SMP presenta mayores requerimientos respecto de la necesidad de asegurar un constante aporte hídrico durante el primer año, intensificar los cuidados culturales, en particular los desmalezados, debido a que presentan mayor susceptibilidad al estrés durante la primera fase del cultivo. El desarrollo radical es un componente clave para el crecimiento de las plántulas en contenedor; ya sea para el desarrollo de la arquitectura radical como para la distribución de las raíces en el interior del contenedor. La alteración morfo-fisiológica de la planta se inicia después que el sistema radical ha ocupado completamente el volumen a su disposición: la consecuencia inmediata es un cambio en el metabolismo de la planta, que se traduce en una visible disminución del crecimiento y en una alteración de la morfología aérea y radical. Existen diversos estudios que confirman esta hipótesis como los realizados por Carmi y Heuer (1981), Ruff et al. (1987). Respecto de la densidad, ésta varía según el manejo y el tipo de espárrago producido, ya que la producción como verde admite distinta densidad. Además de los aspectos de manejo de cultivo mencionados, como inicio, densidad, etc, esta alternativa productiva requiere de un manejo cuidadoso de la cosecha, respetando los indicadores de la especie, en particular el grado de cierre de las brácteas, ya que durante la etapa productiva, las altas temperaturas causan la apertura prematura de los extremos de los turiones, haciendo que se ramifiquen a baja altura, volviéndose no comercializables (Ellison 1986).

El manejo de la cosecha tiene una incidencia directa en el número y el peso promedio de los turiones, ya que son las variables determinantes del rendimiento. El tamaño de la yema está positivamente correlacionado con el tamaño del turión. Nichols y Woolley (1985) y Ellison (1986), trabajando con las mismas plantas durante tres años consecutivos, observaron que los máximos rendimientos provinieron de plantas que tenían muchos turiones grandes.

Una de las variables más importantes durante dicha etapa es la temperatura, cuya influencia varía según se trate de espárrago blanco o verde. Es así que en el manejo verde la inducción de las yemas está determinada por el aumento de temperatura del suelo (Drost 1997), mientras que el crecimiento de los turiones depende fundamentalmente de la temperatura del aire (Krarup y Krarup 1987), ya que la mayor velocidad de crecimiento se ubica por debajo del extremo apical del turión (Keuls y Post 1957.). Existe concordancia entre diferentes autores en que las tasas de elongación diaria de los turiones aumentan entre temperaturas mínimas de 7 a $10^{\circ} \mathrm{C}$ hasta máximas de 25 a $30^{\circ} \mathrm{C}$, y que dichas tasas aumentan también a medida que el turión es mas largo (Blumenfield et al. 1961, Nichols y Woolley 1985, Dean 1999). Debido a la evolución de la temperatura desde el inicio del ciclo productivo, se pueden identificar tres etapas: la primera de crecimiento exponencial (de aproximadamente dos semanas), la segunda de crecimiento continuo (de aproximadamente un mes) y la tercera descendente (de un mes), por el agotamiento de las reservas. Algunos autores han estudiado cada una de las mencionadas etapas el crecimiento de los turiones en función del incremento de la temperatura. (Blumenfield et al. 1961) y calcularon, mediante análisis de regresión, que aumentos de temperatura de $1^{\circ} \mathrm{C}$ generaba aumentos de $0,57 \mathrm{~cm}$ en las tasas de elongación diaria, mientras que aumentos de $1 \mathrm{~cm}$ en la longitud del turión significaban aumentos de $0,35 \mathrm{~cm}$ en las mismas tasas. (Krarup y Mann 1997) reportaron tasas de elongación 
de 5,6; 7,4; y 7,7 cm por día, en turiones de más de 10 $\mathrm{cm}$, asociadas a temperaturas medias de 15,$5 ; 16,6 \mathrm{y}$ $19,8^{\circ} \mathrm{C}$, respectivamente.

El presente trabajo tiene por objetivo determinar la influencia del tamaño de la celda utilizada para la obtención de plántulas de espárrago y la densidad o marco de plantación empleado, sobre la producción comercial de turiones.

\section{MATERIALES Y MÉTODOS}

Este trabajo se inició a mediados de octubre de 2002 en la Chacra Experimental de la Facultad de Agronomía de la Universidad Nacional del Centro de la Provincia de Buenos Aires (UNCPBA), sobre la Ruta Nacional $\mathrm{N}^{\circ} 3, \mathrm{~km} 305$ (a $36^{\circ} 48^{\prime}$ lat. Sur y 59 ${ }^{\circ} 51^{\prime}$ longitud occidental), en el partido de Azul, Provincia de Buenos Aires, sobre un suelo argiduol típico, a través del trasplante de plántulas con cepellón que habían sido sembrados el día 2 de agosto del 2002.

La preparación del lote consistió en dos cinceladas cruzadas, dos pasadas de rastra de discos y una de motocultivador para el refinado de la capa superior. Previo al trasplante se abrieron surcos a una profundidad de $25 \mathrm{~cm}$ con motocultivador equipado con aporcador. Se fertilizó en bandas de $0,30 \mathrm{~m}$ con fosfato diamónico. La dosis utilizada fue de $300 \mathrm{~kg} / \mathrm{ha}$. Se incorporó el fertilizante con un rastrillo, luego se trasplantó manualmente y se efectuó el primer riego.

Se realizó un control de malezas químico, manual y mecánico durante el año de implantación y los siguientes cuatro años. Se utilizó linurón a razón de 2 1/ha en preemergencia del cultivo y de las malezas. Posteriormente se utilizaron otros agroquímicos como metribuzin y pendimetalin a razón de 2 1/ha. Las limpiezas mecánicas se efectuaron mediante el empleo de motocultivador. En la hilera se realizaron controles manuales a lo largo del periodo estival. Luego de efectuada la plantación el ensayo fue dejado vegetar durante los primeros años a fin de propiciar un adecuado desarrollo del aparato radical, por lo que la presente corresponde a la segunda evaluación productiva efectuada en el año 2006.

Etapa de producción de los plántulas: La producción de plántulas se realizó en un invernadero ubicado en la Facultad de Agronomía-UNCPBA, en Azul,Argentina.Las semillasutilizadascorrespondieron a la variedad UC157 F2, fueron sembradas en bandejas de poliestireno expandido esterilizadas, sobre substrato vermiculita, también esterilizado, con tres tamaños de celdas 2,3 y $3,5 \mathrm{~cm}^{2}\left(70 \mathrm{~cm}^{3}, 30 \mathrm{~cm}^{3}\right.$ y $\left.20 \mathrm{~cm}^{3}\right)$.

Una vez sembrados, las bandejas de siembra fueron colocadas en el interior del invernadero de $3,5 \mathrm{~m}$ de altura, con techo semicircular y provisto de ventilación frontal y lateral. Las bandejas de siembra fueron acondicionadas sobre una mesada enrejada a una altura de $1,10 \mathrm{~m}$ para no permitir la salida de las raíces por los orificios de drenaje. El riego se efectuó mediante un sistema abierto sin recuperación de la solución nutritiva.

El trasplante se efectuó a los setenta días con base en un diseño completamente al azar con los tres tratamientos basados en bandejas de siembra: 1) PG: celda grande $\left.\left(70 \mathrm{~cm}^{3}\right), 2\right) \mathrm{PM}$ : celda mediana $\left(30 \mathrm{~cm}^{3}\right)$ y 3) PCh: celda pequeña $\left(20 \mathrm{~cm}^{3}\right)$ y tres densidades D1: 35 714; D2: 23810 y D3 17857 plantas/ha. Las plántulas se plantaron en surcos distanciados a $1,4 \mathrm{~m}$ y con una distancia entre planta de 0,$20 ; 0,30$ y 0,40 $\mathrm{m}$ correspondientes a las mencionadas densidades. $\mathrm{La}$ superficie total fue de $420 \mathrm{~m}^{2}$.

Precipitaciones y temperatura: Durante el año de estudio (2006), correspondiente al segundo período de cosecha, las precipitaciones en la Chacra Experimental fueron de $847 \mathrm{~mm}$. Durante la temporada de cosecha se registraron $31,5 \mathrm{~mm}$ en el mes de septiembre y 162 $\mathrm{mm}$ en octubre, un promedio de $25 \mathrm{~mm}$ semanales, que es un valor aceptable para el cultivo (UNCPBA 2006) (Figura 1).

Cosecha: El presente trabajo corresponde al segundo año de evaluación productiva (2006), se realizaron diecisiete cosechas en el período: $25 / 09$ al 18/10. La frecuencia de cosecha utilizada fue de día por medio. La duración del período de cosecha fue programado en función de la edad de la esparraguera y del desarrollo vegetativo que la misma lograra durante el período vegetativo estival precedente. Se analizó la incidencia de las precipitaciones y temperaturas en las cosechas logradas (Figura 1).

Evaluaciones efectuadas: Las determinaciones realizadas fueron: PF: peso fresco neto, NT: número de turiones por surco y distribución de calibres (C). Fue utilizada la clasificación de turiones de espárragos verdes establecida por el Protocolo de Calidad de Espárragos (SAGPyA 2007) según el cual, el tamaño de los turiones (tomado a $2 \mathrm{~cm}$ desde la base) es 


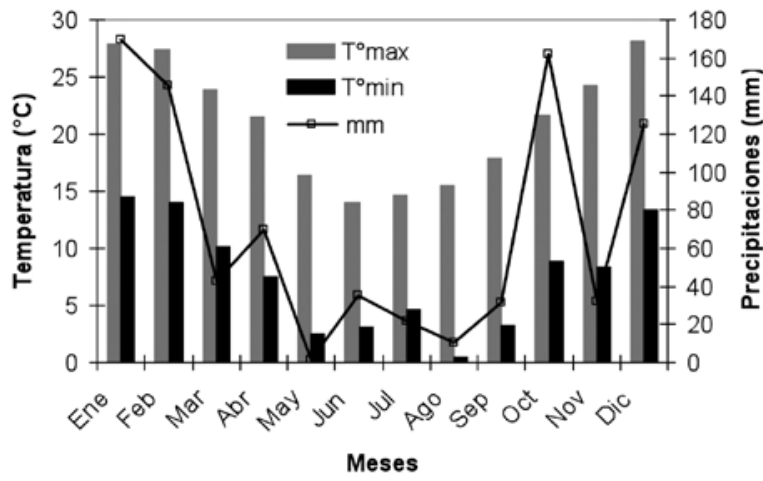

Figura 1. Precipitaciones mensuales $(\mathrm{mm})$ y las temperaturas $\left(\mathrm{T}^{\circ}\right)$ máximas y mínimas, durante el período de estudio. Azul, Provincia de Buenos Aires, Argentina. 01/01/06 - 31/12/06.

determinado por la longitud y/o el diámetro ecuatorial del turión, esto permite la clasificación en diversos calibres (diámetro a $2 \mathrm{~cm}$ desde la base): Small (6$9 \mathrm{~mm})$, Medium $(9-12 \mathrm{~mm})$ y Large $(12-16 \mathrm{~mm})$, clasificación en distintos calibres 1 . S: 6-9 mm; 2 . M: 9-12 mm; 3. L: 12-16 mm; 4. XL: 16-18 mm; 5. Jumbo: $>18 \mathrm{~mm}$. La tolerancia de calidad permitida es de un $10 \%$ de la cantidad o peso del atado, siendo aceptada una diferencia máxima de $2 \mathrm{~mm}$.

Los turiones cosechados fueron lavados y cortados a $22 \mathrm{~cm}$, se descartaron los abiertos y defectuosos.

Para las determinaciones de peso se utilizó una balanza electrónica.

Para las variables en estudio, se realizó el análisis de la varianza (ANOVA) de tipo factorial a dos vías, considerando como factores el tamaño de las plántulas y las densidades. Las medias fueron separadas con el test de la mínima diferencia significativa (LSD) para $\mathrm{P} \leq 0,05$.

\section{RESULTADOS Y DISCUSIÓN}

La cosecha promedio durante el segundo año de evaluación, para el período considerado, fue de 3896 , siendo el promedio por cosecha $229,18 \pm 6,86$ (Cuadro 1).

\section{Tamaño de la celda de la bandeja de siembra}

Respecto de la influencia del tamaño de las celdas en el desarrollo de las plántulas durante el cuarto año desde la plantación y segundo de la evaluación, se
Cuadro 1. Efecto del tamaño de la celda en que se sembraron las plántulas sobre el peso fresco y número de turiones de espárragos verdes (variedad UC157 F2) iniciados por plántulas de distintos tamaños. Azul, Provincia de Buenos Aires, Argentina. 2006.

\begin{tabular}{cccc}
\hline $\begin{array}{c}\text { Tamaño de } \\
\text { celda }\left(\mathbf{c m}^{3}\right)\end{array}$ & $\begin{array}{c}\text { Número de } \\
\text { turiones }\end{array}$ & $\begin{array}{c}\text { Peso fresco } \\
(\mathbf{k g} / \mathbf{h a})\end{array}$ & $\begin{array}{c}\text { Peso prome- } \\
\text { dio/turión }(\mathbf{g})\end{array}$ \\
\hline 70 & $17833,3 \mathrm{~b}^{*}$ & $226,819 \mathrm{ab} *$ & 12,71 \\
30 & $22284,3 \mathrm{a}$ & $253,873 \mathrm{a}$ & 11,39 \\
20 & $21235,3 \mathrm{a}$ & $206,848 \mathrm{~b}$ & 9,74 \\
\hline
\end{tabular}

* Medias seguidas por letras distintas, difieren entre sí según el LSD test $(\mathrm{P}>0,05)$.

lograron mejores resultados con los dos tamaños mayores de los tres considerados, posiblemente debido a los efectos de la restricción radical que sufrieron las plantas provenientes de plántulas con celdas con tamaño de $20 \mathrm{~cm}^{3}$ (PCh). El tamaño de plantín también tuvo incidencia directa en la producción total en $\mathrm{kg}$, los dos mayores tamaños de plántulas G 226,82 (ab) y M 253,88 (a) superaron significativamente a $\mathrm{CH} 206,85$ (b) si bien en cuanto al número de turiones $\mathrm{M}$ y $\mathrm{CH}$.

Con el empleo de plántulas pequeñas (celda de 20 $\mathrm{cm}^{3}$ ) se logró una media inferior al promedio general del ensayo en $23 \mathrm{~kg}$, lo que representa $391 \mathrm{~kg}$ menos para todo el período. En el caso de los tamaños PG y PM superaron al PCh en 47 y $20 \mathrm{~kg}$ respectivamente (Cuadro 1).

Las plantas sometidas a restricción radical (PCh) que habían tenido un mayor desarrollo del aparato radical respecto de la parte aérea, transcurrido aún cinco años, siguen sufriendo los efectos de dicha restricción tal como lo encontrado por Mugnai (1998) en otras especies hortícolas como tomate y tagetes. En este caso produjeron turiones de $9,74 \mathrm{~g}$, es decir $30 \%$ más pequeños que los provenientes de PG y $15 \%$ menores respecto de PM.

Las dos variables estudiadas NT y PF permitieron comprobar que las plantas provenientes de PG produjeron turiones más grandes de $12,71 \mathrm{~g}$ seguidos de PM: $11,39 \mathrm{~g}$.

En el caso de PG se pudo comprobar que si bien el $\mathrm{N}^{\mathrm{o}}$ de turiones resultó menor que en el tamaño de celda PM; el peso fresco (PF) también superó a PCh en $20 \mathrm{~kg}$ promedio/cosecha representando una diferencia de aproximadamente $350 \mathrm{~kg}$ para todo el período de evaluación. 
En el caso de las plántulas grandes para la variable NT, el comportamiento podría ser debido a la presencia de yemas de mayor tamaño respecto de los PM y $\mathrm{PCH}$, lo que influiría sobre el número de turiones totales producidos (Cuadro 1).

\section{Densidad}

La mayor densidad considerada $\left(D_{1}\right)$ fue la que permitió lograr superior rendimiento tanto en peso (4450 kg/ha netos totales) como en número (380 166 turiones) superando a $\mathrm{D}_{2}$ y $\mathrm{D}_{3}\left(\mathrm{D}_{1}: 262(\mathrm{a}) ; \mathrm{D}_{2}: 219\right.$ (b) y $\mathrm{D}_{3}: 207$ (b) kg por cosecha, respectivamente (Cuadro 2). Posiblemente esto se debió a que se trata de una plantación joven que aún no manifiesta competencia intraespecífica (Cuadro 2).

Cuadro 2. Efecto de las densidades sobre el peso fresco y número de turiones de espárragos verdes (variedad UC157 F2) iniciados por plántulas a distinta densidad de plantación. Azul, Provincia de Buenos Aires, Argentina. Setiembre a octubre, 2006.

\begin{tabular}{ccc}
\hline $\begin{array}{c}\text { Densidad } \\
\text { (plantas/ha) }\end{array}$ & $\begin{array}{c}\text { Número } \\
\text { turiones }\end{array}$ & Peso fresco/cosecha \\
\cline { 3 - 3 } & $22362,7 \mathrm{a}^{*}$ & $\mathbf{k g} / \mathbf{h a}$ \\
\hline 35714 & $19147,1 \mathrm{~b}$ & $219,08 \mathrm{~b}$ \\
23810 & $19843,1 \mathrm{~b}$ & $206,72 \mathrm{~b}$ \\
\hline
\end{tabular}

* Medias seguidas por letras distintas, difieren entre si según el LSD test $(\mathrm{P} \leq 0,05)$.

\section{Evolución de la producción a lo largo de las suce- sivas cosechas}

Acorde con la evolución de la temperatura y las precipitaciones, se produjeron picos productivos por tratarse de un cultivo de secano, se destacaron las cosechas ocho y trece para peso fresco $(\mathrm{kg} / \mathrm{ha})$, mientras que para el número de turiones, el mayor valor se obtuvo en la cosecha 13 (Cuadro 3 ) en correspondencia con las precipitaciones (Figura 1).

\section{Distribución de calibres}

La mayor proporción para las distintas variables estudiadas y para todo el periodo considerado
Cuadro 3. Evolución por cosecha del número turiones y peso fresco en espárragos verdes iniciados por plántulas (variedad UC157 F2). Azul, Provincia de Buenos Aires, Argentina. Setiembre a octubre, 2006.

\begin{tabular}{|c|c|c|}
\hline $\begin{array}{c}\text { Cosecha* } \\
\mathrm{N}^{\circ} \\
\end{array}$ & $\begin{array}{c}\text { Número de } \\
\text { turiones/cosecha }\end{array}$ & $\begin{array}{c}\text { Peso fresco } \\
\left(\mathrm{kg} / \mathrm{ha}{ }^{*} \operatorname{cosecha}\right)\end{array}$ \\
\hline 1 & 14222,2 def** & 138,3 fgh $* *$ \\
\hline 2 & 8388,8 & 158,7 efg \\
\hline 3 & 5944,4 & $123,3 \mathrm{gh}$ \\
\hline 4 & $29222,2 \quad b$ & $375,8 \mathrm{ab}$ \\
\hline 5 & 10333,3 ghi & 198,0 defg \\
\hline 6 & $15944,4 \mathrm{de}$ & 180,8 defg \\
\hline 7 & $31722,2 \quad b$ & $240,8 \mathrm{~cd}$ \\
\hline 8 & $18222,2 \mathrm{~cd}$ & 405,2 a \\
\hline 9 & 3637,9 & $74,4 \quad h$ \\
\hline 10 & $31833,3 \quad b$ & $356,1 \mathrm{ab}$ \\
\hline 11 & $26888,9 \quad b$ & 315,8 bc \\
\hline 12 & $19388,9 \mathrm{~cd}$ & $215,2 \mathrm{de}$ \\
\hline 13 & $77944,4 \quad$ a & $343,3 \mathrm{ab}$ \\
\hline 14 & $21388,9 \quad c$ & 196,6 defg \\
\hline 15 & 8111,11 hi & 142,2 efgh \\
\hline 16 & 12722,2 efg & $217,5 \mathrm{de}$ \\
\hline 17 & 15388,9 def & $213,3 \mathrm{def}$ \\
\hline
\end{tabular}

* Cosecha efectuada del 25 de setiembre al 18 de octubre con una frecuencia de día por medio.

** Medias seguidas por letras distintas, difieren entre sí según el LSD test $(\mathrm{P} \leq 0,05)$.

correspondió a los calibres Small y Medium debido a la escasa edad de la plantación.

Calibre XL: sólo en cinco de las diecisiete cosechas efectuadas se pudo observar la presencia de turiones con el máximo grosor, posiblemente debido a las características del híbrido UC157 utilizado para obtener una mayor proporción de bajos calibres y a la edad de la plantación aún joven al momento de la evaluación.

Calibre L: la presencia de este calibre fue mucho más homogénea y solo en dos de las cosechas efectuadas no se produjeron turiones L (Cuadro 4).

\section{Influencia de la densidad sobre la distribución de calibres}

Con la mayor distancia entre plantas $\left(D_{1}\right)$ se observó mayor presencia de calibre M observándose 
Cuadro 4. Efecto del período de cosecha sobre el calibre de turiones de espárragos verdes (variedad UC157 F2) iniciados por plántulas. Azul, Provincia de Buenos Aires, Argentina. Setiembre a octubre, 2006.

\begin{tabular}{|c|c|c|c|c|c|c|}
\hline \multirow[t]{2}{*}{ Cosecha* } & \multicolumn{6}{|c|}{ Calibre $^{* * *}$} \\
\hline & XL & $\mathbf{L}$ & M & $\mathbf{S}$ & VS & Asparagina \\
\hline 1 & $\mathrm{nd}^{* * *}$ & 0,5 bcd $* * * *$ & $2,38 \mathrm{fgh}$ & $4,0 \mathrm{gh}$ & $6,95 \mathrm{de}$ & nd \\
\hline 2 & nd & $0,11 \mathrm{~cd}$ & $2,38 \mathrm{fgh}$ & $2,55 \mathrm{gh}$ & $3,33 \mathrm{fg}$ & nd \\
\hline 3 & nd & nd & $0,56 \mathrm{i}$ & $2,66 \mathrm{gh}$ & $2,5 \mathrm{fg}$ & nd \\
\hline 4 & nd & $0,78 \mathrm{bc}$ & $6,22 a b c$ & 8,83 cde & $13,39 \mathrm{c}$ & nd \\
\hline 5 & $0,056 \mathrm{ab}$ & $0,166 \mathrm{~cd}$ & $2,22 \mathrm{gh}$ & $3,72 \mathrm{gh}$ & 4,16 ef & nd \\
\hline 6 & nd & $0,11 \mathrm{~cd}$ & 3,44 efg & $4,44 \mathrm{fgh}$ & $7,94 \mathrm{~d}$ & nd \\
\hline 7 & nd & $0,11 \mathrm{~cd}$ & 4,0 ef & $9,89 \mathrm{~cd}$ & $17,72 \mathrm{~b}$ & nd \\
\hline 8 & nd & $0,33 \mathrm{bcd}$ & $7,33 a b$ & $12,78 \mathrm{~b}$ & $17,39 \mathrm{~b}$ & nd \\
\hline 9 & $1,11 \mathrm{ab}$ & $0,88 \mathrm{~b}$ & $1,28 \mathrm{hi}$ & $2,55 \mathrm{gh}$ & nd & 1,84 \\
\hline 10 & nd & nd & $7,83 \mathrm{a}$ & $11,05 \mathrm{bc}$ & $13,11 \mathrm{c}$ & nd \\
\hline 11 & nd & $1,72 \mathrm{a}$ & $7,44 \mathrm{ab}$ & $6,95 \mathrm{e}$ & $10,22 \mathrm{~cd}$ & nd \\
\hline 12 & nd & $0,33 \mathrm{bcd}$ & $4,88 \mathrm{cde}$ & 6,55 ef & 7,61 de & nd \\
\hline 13 & nd & $0,78 \mathrm{bc}$ & $5,88 \mathrm{bcd}$ & $17,5 \mathrm{a}$ & 53,77 a & nd \\
\hline 14 & nd & $0,27 \mathrm{bcd}$ & $5,66 \mathrm{~cd}$ & 7,94 de & $7,50 \mathrm{de}$ & nd \\
\hline 15 & $0,056 \mathrm{ab}$ & $0,88 \mathrm{~b}$ & $4,55 \mathrm{de}$ & $2,16 \mathrm{~h}$ & $0,44 \mathrm{~g}$ & nd \\
\hline 16 & $0,056 \mathrm{ab}$ & $2,05 \mathrm{a}$ & $5,83 \mathrm{bcd}$ & $4,17 \mathrm{gh}$ & $0,61 \mathrm{~g}$ & nd \\
\hline 17 & $1,67 \mathrm{a}$ & $2,11 \mathrm{a}$ & $6,33 a b c$ & $4,55 \mathrm{fg}$ & $2,22 \mathrm{fg}$ & nd \\
\hline
\end{tabular}

* Cosecha efectuada del 25 de setiembre al 18 de octubre con una frecuencia de día por medio.

** XL: Extra Large, L: Large, M: Medium, S: Small, VS: Very Small y As: Asparagina.

*** nd: no se registran datos.

**** En columnas, medias seguidas por letras distintas, difieren entre sí según el LSD test $(\mathrm{P}>0,05)$. $(\mathrm{n}=18)$.

diferencias estadísticamente significativas respecto a las otras dos densidades estudiadas, mientras que no se registraron diferencias estadísticamente significativas respecto al factor densidad para los calibres XL y L. En el caso de Asparagina, por tratarse de un calibre indicador de fin de cosecha ante la aparición del mismo se procedió a suspenderla, a fin de preservar la vida útil de la esparraguera.

\section{Influencia del tamaño del plantin sobre la distribu- ción de calibres}

Para los calibres XL, S y Asparagina no se registraron diferencias significativas para el factor tamaño de plantín. En L y M se registraron diferencias estadísticamente significativas entre los factores confrontados para dichos calibres. En efecto, ambos presentaron mayores valores cuando provenían de PG. No existieron diferencias significativas entre PM y PCh.

El tamaño VS se presentó en mayor proporción en los PM y la menor correspondió a los plántulas grandes indicando que el tamaño de plántulas y de yemas influyó en la proporción de turiones VS, en XL y SM se logró similar proporción (Figura 2).

El tamaño de plántula utilizado en un cultivo perenne como el espárrago, mostró una incidencia directa en el rendimiento logrado en peso fresco como en $\mathrm{n}^{\circ}$ de turiones cosechados, indicando la importancia en la elección del tamaño a utilizar.

Las bandejas de siembra de tamaño grande resultan más apropiadas para la producción de plántulas de espárrago ya que permitieron obtener un mejor 


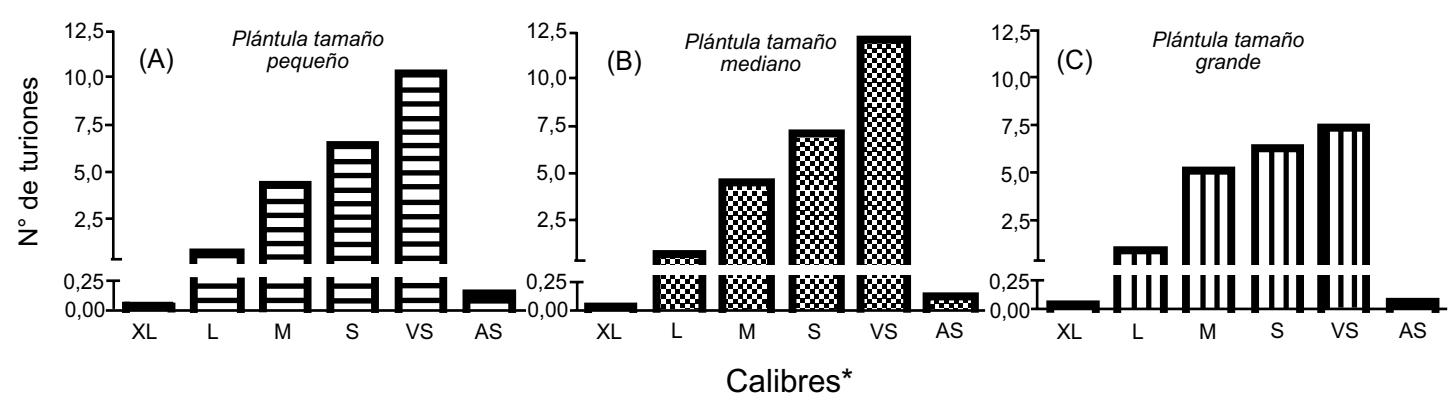

Figura 2. Calibres o grosor de los turiones obtenidos en una plantación de espárrago verde mediante el inicio del cultivo con distintos tamaños de plántulas inducido por el empleo de bandejas de diferente tamaño de celda. Azul, Provincia de Buenos Aires, Argentina. Setiembre a octubre, 2006.

* Calibre XL (Extra Large); L (Large); M (Medium); S (Small); VS (Very Small) y As (Asparagina).

desarrollo radical, aspecto fundamental por tratarse de un cultivo perenne (Castagnino et al. 2002). Las plantas sometidas a restricción radical en las bandejas de siembra mayormente aireadas resultaron mejor.

Considerando que el ensayo corresponde a un cultivo productivo y con un manejo de secano, de las tres fases en que puede subdividirse la cosecha, se procedió a efectuar la recolección en las dos primeras: la inicial a los diez días, la temperatura representó el factor limitante de la cantidad de turiones producidos. La intermedia a los treinta días, cuando el factor que condiciona el rendimiento no es más la temperatura y sí la capacidad del híbrido. No se cosechó en una fase final, ya que la misma se caracteriza por una reducción progresiva de cada cosecha, ya sea de la producción como del calibre de los turiones (Falavigna y Palumbo 2001).

Afectaron el rendimiento tanto la densidad como el inicio del cultivo con plántulas de distintos tamaños producidos con restricción del volumen a disposición de las raíces.

La restricción del volumen a disposición de las raíces en la etapa de vivero causó alteraciones significativas en el crecimiento y desarrollo de las plantas en cultivo definitivo, dando rendimientos menores tanto en número como en $\mathrm{kg} / \mathrm{ha}$ y en el tamaño de los turiones producidos. Muchos autores sostienen la hipótesis que la reducida capacidad fotosintética de las plantas estresadas como causa de su reducción del crecimiento, demuestran como una disminución de la asimilación fotosintética neta sea uno de los más evidentes efectos del estrés ocasionado por la restricción radical (Mugnai 1998). Por tal motivo, estos resultados indican la importancia de utilizar bandejas de siembra provistas de tamaño de celda adecuado a la especie.

El inicio de una esparraguera mediante el empleo de plántulas posibilita la programación adecuada del transplante y el logro de turiones más uniformes y mayor calidad.

Las bandejas de siembra de tamaño grande resultan más apropiadas para la producción de plántulas de espárrago ya que permiten obtener un mejor desarrollo radical, aspecto fundamental por tratarse de un cultivo perenne y evitar la restricción radical en una etapa vital del cultivo.

Para una plantación joven resultó más conveniente el empleo de la mayor densidad $(4450 \mathrm{~kg} /$ ha netos totales y 380166 turiones). El tamaño de plantín también tuvo incidencia directa en la producción total en $\mathrm{kg}$.

Para la producción de turiones de calibre grande, influyó el mayor tamaño de plantín, mientras que cuando el objetivo es producir turiones de calibres bajos, no es tan importante el tamaño de la bandeja (Figura 2).

Sería necesario continuar estudiando la incidencia del tamaño de plantín utilizado en la producción de turiones cosechados a lo largo de las sucesivas cosechas.

\section{LITERATURA CITADA}

Blumenfield, DK; Meinken, KW; Le Compte,SB 1961. A field study of asparagus growth. Proc. Am. Soc. Hortic. Sci. 77:386-392. 
Carmi, A; Heuer, B. 1981. El papel de las raíces en el control del crecimiento de frijol disparar. Ann. Bot. 48:419527.

Castagnino, AM; Sastre Vázquez, P; Diaz, K. 2002. Efecto del contenedor sobre la calidad de plantines de espárrago IX Congreso Nacional y II Internacional de Horticultura Ornamental. Asociación Mexicana de Horticultura Ornamental y la Sociedad Mexicana de Ciencias Hortícolas, México (en línea). Consultado dic. 2008. Disponible en http://www.chapingo.mx/Fitos/eventos/congresos2003/congresos2003.html

Dean, BB. 1999. The effect of temperature on asparagus spear growth and correlation of heat units accumulated in the field with spear yield. In Benson B. ed. Proc. $9^{\text {th }}$ Int. Asparagus Symp. Acta Hortic. 479:289295.

Drost, DT. 1997. Asparagus. In Wien, HC. ed. The physiology of vegetable crops. CAB Internacional. Wallingford, New York. p. 621-649.

Ellison, JH. 1986. Asparagus breeding. In Basset, MJ. ed. Breeding vegetables crops. Wesport: AVI. p. 521-569.

Falavigna, A, Palumbo, AD. 2001. La coltura del aspárago. Calderini Edagricole. Italia. p. 51.

Keuls, M; Post, JJ. 1957. Invloed van de temperatuur op de groi van asperges. Overdruk uit de Mededelingen Directeur Tuinbouw 19:827-845.
Krarup, A; Mann, D. 1997. Elongación diaria y altura de apertura de la cabeza de los turiones de veintiocho genotipos de espárrago. Agro Sur 25:16-23.

Krarup, AH; Krarup, JPL. 1987. Rendimiento de espárragos verdes y blancos bajo dos modalidades de cosecha. Agro Sur 15:47-53.

Mugnai, S. 1998. Restrizione Radicale: Efetti Morfo-fisiologici in piantine di pomodoro (Lycopersicon esculentum Mill) e Tagete (Tagetes patula L.), Tesis de Dotorado de investigación in Ortoflorofrutticoltura. U. degli Studi di Pisa, Italia.

Nichols, MA; Woolley, D. 1985. Growth studies with asparagus. International Asparagus Symposium, 6, Proceedings. Ontario: University of Guelph. p. 287-297.

Ruff, MS, Krizek, DT, Mirecki, RM; Inouye, DW. 1987. Restringida volumen zona de la raíz: influencia sobre el crecimiento y el desarrollo de tomate. J. Amer. Soc. Hort. Sci. 112:736-739.

SAGPyA (Secretaría de Agricultura, Ganadería, Pesca y Alimentos). 2007. Protocolo de calidad para espárrago fresco. Resolución SAGPyA Nº 249/2007. http://www. alimentosargentinos.gov.ar/programa_calidad/diferenciacion/sello/SAA010_Esparrago_v08.pdf

UNCPBA (Universidad Nacional del Centro de la Provincia de Buenos Aires). 2006. Boletín Agrometeorológico. Datos de temperatura. 